\title{
Model Comparison and Data Interface Program Development between BPA and PSS/E
}

\author{
Peng Li, Peng Tian, Hezhi Liu, Xu Wei \\ Jiangjin Power Supply Bureau, Chongqing Electric Power Company, Chongqing, 402260, China \\ E-mail: liuhezhi@yeah.net
}

\begin{abstract}
Write a data interface program transforming data between BPA and PSS/E by VC and realize bidirectional transform of power flow data and transition from BPA to PSS/E of stable data. Illuminate the corresponding relationship between these two programs of both power flow and stable data and compare differences and resemblances between them. Emphasize the difference in dealing with excitation and governor model; explain the essential points during transform and measurements of managing discrepant model. Validate the effectiveness of this program by simulation.
\end{abstract}

Keywords-BPA; PSS/E; Interface Program; Power Flow; Stability; PSS

\section{INTRODUCTION}

At present we mainly use PSASP (developed by China Electric Power Research Institute) and BPA to calculate the grid simulation. While PSS/E (developed by PTI Company) has more powerful function, it can not only calculate the steady and transient simulation, but also use programming to realize complex function and use the mathematical model which PSS/E provides to expand through the user-defined way. The data of BPA and PSASP can use the PCS digital simulation platform (developed by China Electric Power Research Institute) to transform its data file format to each other, and PSS/E also provides IEEE data format conversion. Through the IEEE format, we can share the data in foreign other analysis software. But it is a pity that BPA and PSS/E have no mature conversion program now, the data file cannot be shared between them. Thus if we want to use foreign software powerful analysis function, we need to make the cumbersome data entry work which is huge workload and belongs to the repetition labor. In order to solve this problem, many workers have had on the part of the interface program development. They have compare the model partly in each other while there is no systematically compare their similarities and differences between them, and does not compare the excitation, speed control system models (References [1-3]).

In this paper, we compare the data format and model between BPA and PSS/E, and use VC programming to realize the data file conversion. It establishes the bridge between the domestic software and foreign software, and provides great convenience to system analyst. Finally, the simulation results show the effectiveness of the interface program.

\section{INTERFACE PROGRAM'S STRUCTURE}

Interface program's structure and its relationship between input and output are shown in figure 1 and 2.

This program finishes the power flow's data transfer between BPA and PSS/E, and realizes the stability data transfer from BPA to PSS/E. After that, we detailed compare the steady state and transient mathematical model between BPA and PSS/E, and show the matters that we need attention in the transformation process.

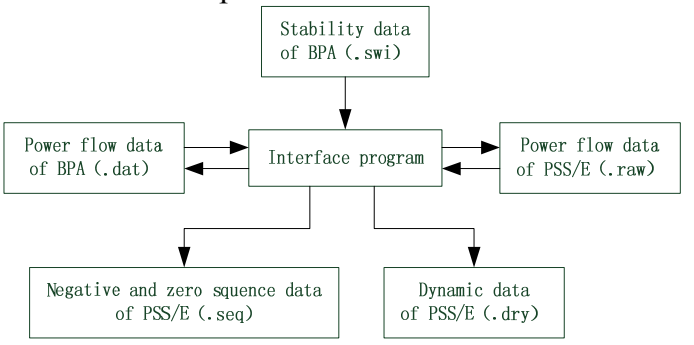

Figure 1. Relationship diagram of the program

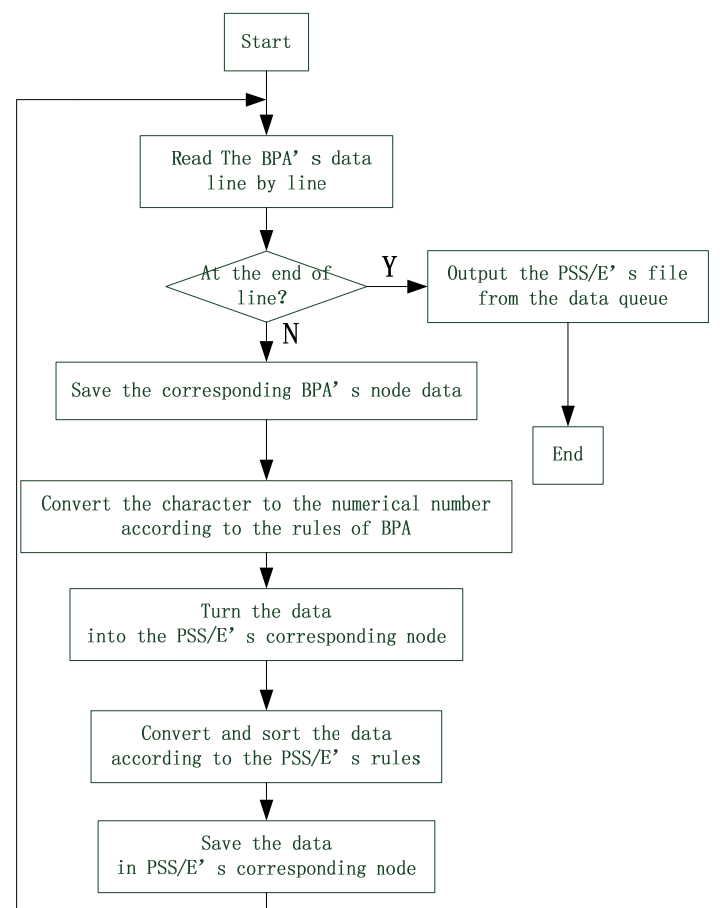

Figure 2. Diagram of program's flow 


\section{MODEL COMPARE OF INTERFACE PROGRAM}

\section{A. Compare of power flow's data between BPA and PSS/E}

According to the bus data, BPA's bus data contains the generator data, load data, while these three types of data need fill in separately in PSS/E; BPA's node types have a clear rules and do not support the isolated bus, and PSS/E's node types have load bus, generator or power plant node, balance nodes and isolated bus.

The corresponding relations of node types are shown in TABLE I.

According to the branch data, asymmetric branches are filled by the $\mathrm{L}$ card and $\mathrm{E}$ card in BPA, while the symmetrical and asymmetric branches are unified to fill in PSS/E. according to this, we can have two ways to process, one is two cards (L Card and E Card) merger processing, the other is making the asymmetric data as a shunt admittance processing after artificial increasing bus.

According to two winding transformer, reference [3] points out that BPA uses variable ratio's adjustable model between high and low voltage side, while PSS/E uses variable ratio's adjustable model of low voltage side. This point in PSS/E30 has already got unification, which adopted variable ratio's adjustable model between high and low voltage side. Reference [6] gives the processing method for OLTC (On-Load Tap Changer) and the parameter analysis between BPA and PSS/E's model. PSS/E supports three winding transformer, and BPA only supports two winding transformer. Therefore, in $\mathrm{PSS} / \mathrm{E}$ to $\mathrm{BPA}$ conversion, the program should be in three winding transformer center point people to join a bus, three-winding transformer into three two winding transformer, the original three side variable ratio is switched three two winding transformer primary side variable ratio, secondary side variable ratio is 1 . After converting the resistance of the transformer reactance computation formula is as follows:

$$
\left\{\begin{array}{l}
Z_{T 1}=\frac{1}{2}\left(Z_{1-2}+Z_{3-1}-Z_{2-3}\right) \\
Z_{T 2}=\frac{1}{2}\left(Z_{1-2}+Z_{2-3}-Z_{3-1}\right) \\
Z_{T 3}=\frac{1}{2}\left(Z_{2-3}+Z_{3-1}-Z_{1-2}\right)
\end{array}\right.
$$

In this equation, the $\mathrm{Z}$ presents transformer winding's P.U. value of positive sequence resistance or reactance.

According to the generator data, PSS/E turns step-up transformer of factory station into generator node data, while the BPA separately fill in bus data and transformer data. Therefore, when we convert PSS/E to BPA data, we should artificially increase a bus in primary side of step-up transformers. If it involves generator to bus tidal current or voltage control, we need to remote control, control the bus of step-up transformer's primary side.

According to the load data, PSS/E supports three types of load (those are constant power, constant current and constant admittance) in power flow calculation but they don't distinguish. While BPA processes them only as constant power loads. Therefore, we just add the PSS/E's three types of load power together to get the BPA's load data in conversion.

In addition, we should pay attention to the process of space besides BPA's data format rules. In BPA's regulation, if numeric data begins to the space, the program fills zero automatically, and then identifies according to the definition of the rules; if character data begins to the space, it is not do any treatment, while if two character data in addition to the space outside the same other characters, the program still considers them as different data. Thus if we ignores this, it will cause the calculation results of the large deviation, and it is difficult to find out the reasons. To this problem, the program does the special treatment. In order to avoid misunderstanding to fill in the data, we suggest filling in the data top grid.

Through power flow's calculation of a local grid when summer peak, we verify the effectiveness of the interface program. Due to the large amount of data, we list the $500 \mathrm{kV}$ buses' voltage amplitude and phase angle, and balance machine's output information. The results are shown in TABLE II.

From TABLE II we can see that the calculation results satisfy perfectly in large power system's simulation. Meanwhile it shows the interface program in power flow's data conversion is very effective.

\section{B. Compare of stability data between BPA and PSS/E}

1) Generator's dynamic data. Generator model which BPA provides are:

- Subtransient model (biaxial model which considers damping winding).

- Ransient model (biaxial model which doesn't consider damping winding).

- Classical Model $\left(E^{\prime}=C\right)$.

- Equivalent load model (generators which affect small and is not in research range, using LN card to present).

The generator models of PSS/E are more abundant, and it can consider saturation coefficient. TABLE III shows the corresponding relationship of generator model between BPA and PSS/E.

In addition, the PSS/E also provides a full order model of generator (CGEN1). It can simulate generator model in any situation, non-salient pole machine model can use this model. 2) Load model. BPA provides three types of load model, which are static model, induction motor model and comprehensive model considering distribution network's branches. It is through filling in the control and protection data card to realize the low pressure low frequency load reduction model. PSS/E contains more types of models, it not only provides static model and dynamic model, but also provides low pressure low frequency load reduction model. TABLE IV shows corresponding relationship of the load models between BPA and PSS/E.

3) Excitation system's model. At present, no references make comparison of excitation and speed control system's models between BPA and PSS/E. The reason is that there is large 
different between them. In this section, we focus on the common excitation model to compare and process. The corresponding relations of excitation system's models are shown in TABLE V.

TABLE I. BUS NODE TYPE CORRESPONDING RELATIONS

\begin{tabular}{cc}
\hline BPA's bus type & PSS/E's bus type \\
\hline B, BC & Type 1 (PQ or load node) \\
BE, BG, BQ & Type 2 (PV or generator \\
BS & node) \\
\hline
\end{tabular}

TABLE II. COMPARE RESULTS OF 500KV GRID'S POWER FLOW IN AN AREA

\begin{tabular}{|c|c|c|c|c|c|c|}
\hline \multirow[b]{2}{*}{ Name } & \multicolumn{2}{|c|}{ BPA } & \multicolumn{2}{|c|}{$\mathrm{PSS} / \mathrm{E}$} & \multicolumn{2}{|c|}{ Error } \\
\hline & $\begin{array}{c}\mathrm{V} \\
\mathrm{kV}\end{array}$ & $\begin{array}{c}\mathrm{A} \\
\circ\end{array}$ & $\begin{array}{c}\mathrm{V} \\
\mathrm{kV}\end{array}$ & $\begin{array}{c}\mathrm{A} \\
\circ\end{array}$ & $\begin{array}{l}\Delta \mathrm{V} \\
\mathrm{kV}\end{array}$ & $\stackrel{\Delta \mathrm{A}}{\circ}$ \\
\hline DaTang & 523.61 & 23.2 & 523.63 & 23.21 & -0.02 & -0.01 \\
\hline DaYuan & 516.57 & 11.4 & 516.58 & 11.46 & -0.01 & -0.06 \\
\hline DongTai & 521.38 & 17.3 & 521.39 & 17.33 & -0.01 & -0.03 \\
\hline DouShan & 501.96 & 22.1 & 502.17 & 22.07 & -0.21 & 0.03 \\
\hline FuZhou & 523.87 & 19.4 & 523.89 & 19.48 & -0.02 & -0.08 \\
\hline HaiChang & 516.87 & 12.8 & 516.87 & 12.82 & 0.00 & -0.02 \\
\hline HouShi & 525.71 & 17.7 & 525.71 & 17.75 & 0.00 & -0.05 \\
\hline HuiQuan & 495.97 & 23.0 & 496.49 & 22.95 & -0.52 & 0.05 \\
\hline JiangYin & 521.79 & 19.0 & 521.8 & 19.09 & -0.01 & -0.09 \\
\hline JingFeng & 495.68 & 19.8 & 495.85 & 19.77 & -0.17 & 0.03 \\
\hline JinJiang & 513.35 & 10.4 & 513.35 & 10.48 & 0.00 & -0.08 \\
\hline KeMen & 527.4 & 23.5 & 527.41 & 23.57 & -0.01 & -0.07 \\
\hline LianJiang & 523.83 & 19.8 & 523.85 & 19.82 & -0.02 & -0.02 \\
\hline LongWangsan & 509.81 & 29.1 & 510.34 & 29.09 & -0.53 & 0.01 \\
\hline LvSigang & 507.65 & 27.8 & 507.75 & 27.78 & -0.10 & 0.02 \\
\hline LingDe & 523.67 & 21.1 & 523.7 & 21.1 & -0.03 & 0.00 \\
\hline PuTian & 521.17 & 14.2 & 521.18 & 14.21 & -0.01 & -0.01 \\
\hline QianYun & 521.95 & 14.6 & 521.95 & 14.65 & 0.00 & -0.05 \\
\hline QuanZhou & 515.74 & 11.0 & 515.74 & 11.08 & 0.00 & -0.08 \\
\hline SanChawan & 516.92 & 32.2 & 517.41 & 32.18 & -0.49 & 0.02 \\
\hline ShuangSi & 515.93 & 37.7 & 516.08 & 37.67 & -0.15 & 0.03 \\
\hline ShuiKou & 527.78 & 17.6 & 527.79 & 17.61 & -0.01 & -0.01 \\
\hline TaiBei & 502.97 & 24.9 & 503.13 & 24.91 & -0.16 & -0.01 \\
\hline XiaMen & 515.41 & 11.8 & 515.41 & 11.89 & 0.00 & -0.09 \\
\hline YiHua 1 & 497.38 & 15.7 & 497.48 & 15.61 & -0.10 & 0.09 \\
\hline YiHua 2 & 497.38 & 15.7 & 497.48 & 15.61 & -0.10 & 0.09 \\
\hline ZhangZhou & 522.75 & 14.4 & 522.75 & 14.41 & 0.00 & -0.01 \\
\hline SanYang & 533.41 & 16.0 & 533.42 & 15.99 & -0.01 & 0.01 \\
\hline NanPing & 528.97 & 17.5 & 529 & 17.56 & -0.03 & -0.06 \\
\hline ZuoRan & 530.21 & 14.5 & 530.22 & 14.54 & -0.01 & -0.04 \\
\hline $\begin{array}{c}\text { Slack Node } \\
\text { Output }\end{array}$ & $\begin{array}{c}\mathrm{P} \\
\mathrm{MW}\end{array}$ & $\begin{array}{c}\text { Q } \\
\text { MVar }\end{array}$ & $\begin{array}{c}\mathrm{P} \\
\mathrm{MW}\end{array}$ & $\begin{array}{c}\mathrm{Q} \\
\mathrm{MVar}\end{array}$ & $\begin{array}{c}\Delta \mathrm{P} \\
\mathrm{MW}\end{array}$ & $\begin{array}{c}\Delta \mathrm{Q} \\
\mathrm{MVar}\end{array}$ \\
\hline BL1 & -1232.2 & 352.7 & -1232.6 & 352.7 & 0.40 & 0.02 \\
\hline
\end{tabular}

TABLE III. RELATIONSHIP OF GENERATOR'S MODEL BETWEEN BPA AND PSS/E

\begin{tabular}{|c|c|c|c|}
\hline Order & $\begin{array}{c}\text { Winding } \\
\text { considering }\end{array}$ & BPA & $\mathrm{PSS} / \mathrm{E}$ \\
\hline $\begin{array}{l}\text { Subtransient } \\
\text { model in non- } \\
\text { salient pole } \\
\text { machine } \\
\text { (Six order) }\end{array}$ & $\begin{array}{c}\mathrm{f} 、 \mathrm{~g} 、 \mathrm{D} 、 \\
\mathrm{Q}\end{array}$ & $\begin{array}{c}\text { Biaxial } \\
\text { model } \\
\text { consider } \\
\text { damping } \\
\text { winding }\end{array}$ & GENROU \\
\hline $\begin{array}{l}\text { Subtransient } \\
\text { model in } \\
\text { salient pole } \\
\text { machine } \\
\text { (Five order) }\end{array}$ & $f 、 D 、 Q$ & $\begin{array}{l}\text { Biaxial } \\
\text { model not } \\
\text { consider g } \\
\text { winding }\end{array}$ & GENSAL \\
\hline $\begin{array}{l}\text { Transient } \\
\text { model in non- } \\
\text { salient pole } \\
\text { machine } \\
\text { (Four order) }\end{array}$ & $f 、 g$ & $\begin{array}{l}\text { Biaxial } \\
\text { model not } \\
\text { consider } \\
\text { damping } \\
\text { winding }\end{array}$ & NULL \\
\hline $\begin{array}{l}\text { Transient } \\
\text { model in } \\
\text { salient pole } \\
\text { machine } \\
\text { (Three order) }\end{array}$ & $\mathrm{f}$ & $\begin{array}{l}\text { Biaxial } \\
\text { model not } \\
\text { consider } \\
\text { damping } \\
\text { winding }\end{array}$ & GENTRA \\
\hline Two order & NULL & $\begin{array}{c}\text { Classical } \\
\text { model }\end{array}$ & GENCLS \\
\hline
\end{tabular}

TABLE IV. RELATIONSHIP OF THE LOAD MODELS BETWEEN BPA AND $\mathrm{PSS} / \mathrm{E}$

\begin{tabular}{ccr}
\hline Model type & BPA & PSS/E \\
\hline Static load & LA、LB、 L+ & IEELBL、 \\
Induction \\
motor & MI、ML、MJ & CIM5BL、 \\
$\begin{array}{c}\text { Comprehensive } \\
\text { load }\end{array}$ & LE & CIMWBL \\
Low voltage & - & CLODBL \\
load shedding & & LVS3BL、 \\
& & LVSHBL \\
Low frequency & & DLSHBL、 \\
load shedding & - & LDSHBL、 \\
& & LDS3BL、 \\
\hline
\end{tabular}

TABLE V. RELATIONSHIP OF EXCITATION SYSTEM'S MODELS BETWEEN BPA AND PSS/E

\begin{tabular}{ccc}
\hline Model type & BPA & PSS/E \\
\hline Continuous, rotating, DC & EA & IEEEX1 \\
Brushless, rotating, AC & EB & IEEEX2 \\
Brushless, rotating, AC(improved) & EC & IEEX2A \\
Compound excitation & ED & IEEET3 \\
Discontinuous adjustment rheostat & EE & IEET5A \\
Brushless, rotating, AC & EF & - \\
Silicon controlled rectifier & EG & IEEEX1 \\
DC exciter commutator & FA & ESDC1A \\
DC exciter commutator & FB & ESDC2A \\
Uncontrollable rectifier AC generator & FC & EXAC1 \\
- rectifier & &
\end{tabular}




$\begin{array}{ccc}\begin{array}{c}\text { Composite source rectifier } \\ \text { Non continuous regulator DC exciter } \\ \text { commutator }\end{array} & \text { FD } & \text { EXST2 } \\ \begin{array}{c}\text { Fncontrollable rectifier AC generator } \\ - \text { rectifier }\end{array} & \text { FF } & \text { EXAC2 } \\ \text { Alternator rectifier(controllable) } & \text { FG } & \text { EXAC4 } \\ \text { Alternator rectifier } & \text { FH } & \text { EXAC3 } \\ \text { Potential source SCR rectifier } & \text { FJ、 } & \text { EXST1 } \\ \text { Composite source rectifier } & \text { FL } & \text { IEEET1 }\end{array}$

For the SCR excitation system (EG), because it does not consider saturated parameters' influence (i.e. non saturation curve for linear relationship), we make the appropriate treatment in the conversion. In BPA, DC commutator exciter excitation system model does not consider excitation limiting role, while in $\mathrm{PSS} / \mathrm{E}$, corresponding model considers this influence. Therefore, in order to ensure conversion consistent, we engages in excitation limiting link in this program, then sets its magnification factor heavily, after that the transient calculation can ignore the influence of this link.

4) Speed control system model. In PSS/E, speed control system and prime mover are modeling unified, while in BPA, they are modeling separately. Meanwhile, these two kinds of software use different models. The following gives the model on the result of the treatment, and the dynamic mathematical model is shown in References [8] and [9].

After model reduction and comparison, the process between hydraulic turbine governor and prime mover's model $(\mathrm{GH})$ of BPA and IEEEG3 model of PSS/E is as follows:

$$
a_{11}=0.5, \quad a_{13} \cdot a_{21} / a_{23}=1.5, \quad a_{23}=1,
$$$$
\sigma=D_{d} / R+1, \quad T_{R}=T_{D}, \quad \delta_{S}=-D_{d} / R T_{d}, T_{G}=T_{G} / R .
$$

According to models of speeding controller (GS) and prime mover ( $T A 、 T B 、 T C 、 T D 、 T E 、 T F)$, the parameters relations between GS and IEEEG3 are as follows:

TA: $T_{4}=T_{C H}, K_{1}=1$, others equal 0;

TB : $K_{2}=K_{4}=K_{6}=K_{7}=K_{8}=0, K_{1}=(1+\lambda) F_{H P}$, $K_{3}=0.5 F_{I P}, K_{5}=0.5 F_{L P}, T_{4}=T_{C H}, T_{5}=0.5 T_{R H}$, $T_{6}=T_{C O}, T_{7}=0$;

TC : $K_{2}=K_{4}=K_{6}=K_{8}=0, K_{1}=F_{V H P}, K_{3}=F_{H P}$, $K_{5}=F_{I P}, K_{7}=F_{L P}, T_{4}=T_{C H}, T_{5}=T_{R H 1}, T_{6}=T_{R H 2}$, $T_{7}=T_{C O}$;

$T D: K_{1}=F_{H P}, K_{2}=K_{3}=K_{7}=K_{8}=0, K_{4}=F_{L P} / 2$, $K_{6}=F_{L P} / 2, T_{4}=T_{C H}, T_{5}=T_{R H}, T_{6}=T_{C O}, T_{7}=0$;

$T E: K_{1}=F_{V H P}, K_{2}=K_{4}=K_{5}=0, \quad K_{3}=F_{I P}$, $K_{6}=F_{L P}, T_{4}=T_{C H}, T_{5}=T_{R H}, T_{6}=T_{C O}, T_{7}=0$;

$T F: K_{1}=F_{V H P}, K_{2}=K_{3}=0, K_{4}=F_{H P}, K_{5}=F_{I P} / 2$, $K_{6}=F_{I P} / 2, K_{7}=K_{8}=F_{L P} / 2, T_{4}=T_{C H}, T_{5}=T_{R H 1}$, $T_{6}=T_{R H 2}, T_{7}=T_{C O}$.
The above parameters corresponding relation, the left side is PSS/E's parameters, and the right side is BPA's parameters.

5) PSS' model. In the correspondence of the IEE2ST, BPA card's subtype letters and their meanings are as follows:

$F$ : The input is the variation value of bus frequency;

$P:$ The input is the power which is used to speed up;

$S$ : The input is axial slip;

$G$ : The input is electromagnetic power difference.

While in IEE2ST, IC value expressed the input signal, its meaning is as follows:

$I C=1:$ Speed change value;

$I C=2$ : The variation value of bus frequency;

$I C=3$ : Electromagnetic power difference;

$I C=4$ : The power which is used to speed up;

$I C=5$ : Bus voltage;

$I C=6$ : Bus voltage deviation.

In addition, when we make the conversion, we should make the two equals as follows:

$$
V_{C L}=V_{T 0}-V_{\text {CUTOFF }}, V_{C U}=V_{T 0}+V_{\text {CUTOFF }} \text {. }
$$

\section{SUMMARY AND PROSPECT}

This paper compares the mathematical models of power flow and stability calculation between BPA and PSS/E, and points out the differences between them. Then it uses VC to develop the interface program of data conversion. In order to ensure the consistency of these two kinds of software's calculation results, it uses some grid's data to verify the rationality and effectiveness through the simulation. This software has been successfully applied in the calculation of the data conversion in the power grid, and it greatly reduces the data entry work.

Meanwhile, after model comparison, the author finds that there are some stability models cannot find the corresponding ones. We need to use PSS/E's custom module to structure them artificially, and then use this software to convert the data. The author is to study this.

\section{REFERENCES}

[1] Z. Xu, Q.J. Xu, L. Ren. "Comparison of load models for electromechanical transient simulation in BAP, PSASP and PSS/E". Southern Power System Technology, vol. 2(5), pp. 32-35, 2008.

[2] R.J. Zhu. "Applied study of PSS/E general generator model". East China Electric Power, vol. 32(3), pp. 4-7, 2004.

[3] R.J. Zhu. "Study of generator model with PSS/E stability calculation program". East China Electric Power, vol. 32(7), pp. 1-5, 2003.

[4] G.Q. Li. "Transient analysis of power system". Beijing: Water Power Press, 1984.

[5] J.Y. Huang, W.H. Chen. "Synchronous motor basic theory and its dynamic behavior analysis". Shanghai: Shanghai Jiaotong University Press, 1989.

[6] Q.L. Ni, C.L. Lu, Y. Zhang. "Analysis on tap-changing transformers model parameter”. Zhejiang Electric Power, vol. 3, pp. 1-4, 2005.

[7] PSD-BPA user manual of power flow program. China Electric Power Research Institute. 2007. 